\title{
PWE-016 MRI FOR DIAGNOSIS OF SMALL BOWEL DISEASE IN PAEDIATRIC CROHNS DISEASE: A SYSTEMATIC REVIEW
}

doi:10.1136/gut.2011.239301.279

E Giles, ${ }^{1, *}$ A R Barclay, ${ }^{2}$ S Chippington, ${ }^{3}$ D C Wilson 4 'Paediatric Gastroenterology, Royal London Hospital, London, UK; ${ }^{2}$ Paediatric Gastroenterology, Royal Hospital for Sick Children, Glasgow, UK; ' ${ }^{P}$ Paediatric Radiology, Great Ormond Street Hospital, London, UK; ${ }^{4}$ Child Life and Health, University of Edinburgh, Edinburgh, UK

Introduction Barium meal enteroclysis (BM) is the recommended imaging technique for small bowel inaccessable by ileocolonoscopy when diagnosing paediatric inflammatory bowel disease (IBD) (1). However it is subject to poor sensitivity and involves ionising radiation. MRI enterography (MRE) has recently been reported as an alternative methodology. We aimed to critically appraise the published evidence on use of MRE in diagnosis of paediatric IBD by systematic review.

Methods Review of all English language data reporting MRE for the investigation of patients $<16$ years with known or suspected IBD. Searches of Medline (January 1950-November 2010) and Pubmed (January 1950-November 2010) were performed using keyword and MeSH terms; IBD; MRI; small bowel imaging. Reference lists of potential studies, handsearching and personal collections of authors were also

Table 1 PWE-016

\begin{tabular}{|c|c|c|c|c|}
\hline Study & Patient No & Index test & Reference test & Conclusions \\
\hline Dagia 2008 & 24 & MRE & Endoscopy & $7 / 28$ identified small bowel disease \\
\hline Dagia 2010 & 46 & MRE & Endoscopy & $86 \%$ sensitivity \\
\hline Horsthius 2010 & 33 & MRE and BM & Endoscopy & $\begin{array}{l}60-90 \% \text { sensitivity and specificity, } 50-80 \% \text { sensitivity } 63-94 \% \\
\text { specificity for BM }\end{array}$ \\
\hline Diarbari 2004 & 58 & MRE and $\mathrm{BM}, \mathrm{CT}$ & Endoscopy & $\begin{array}{l}96 \% \text { sensitivity } 92 \% \text { specific } \\
14 / 21 \text { proximal small bowel disease, more sensitive than } B M .\end{array}$ \\
\hline Durno 2000 & 14 & MRE & Endoscopy & 5/10 identified extensive small bowel disease. \\
\hline Magnano 2003 & 22 & MRE and US & Endoscopy & $90 \%$ sensitivity, US equivalent \\
\hline Laghi 2003 & 75 & MRE & Endoscopy & $84 \%$ sensitivity $100 \%$ specificity \\
\hline Borthe 2006 & 43 & MRE and US & Endoscopic & $82 \%$ sensitivity $100 \%$ specificity, US equivalent \\
\hline
\end{tabular}


examined. Two authors independently assessed the quality of studies for inclusion using the QUADAS tool (2). A third author was an arbiter in cases of disagreement.

Results Database searches yielded 606291 hits, combination word searches limited this to 968 titles. 38 studies were fully reviewed and 10 potential studies identified. 2 studies were excluded due to lack of separate paediatric data or inadequate methodological rigour. 8 studies were included (QUADAS scores 7-13/14) (table 1). Studies displayed heterogeneity in bowel preparation, scanning technique, reporting methodology and comparisons with BM, ultrasound and CT. Timing of ileocolonoscopy in relation to MRE was also variable. Two papers reported greater sensitivity and specificity for MRE in comparison to BM.

Conclusion MRE is an sensitive and specific tool for the diagnosis of paediatric IBD. However technical considerations require refinement and standardisation, however MRE offers a significant reduction in ionising radiation exposure. Current data suggest that MRE should supercede BM as the small bowel imaging technique in centres with appropriate expertise.

\section{Competing interests None.}

Keywords inflammatory bowel disease, paediatric, small bowel imaging.

\section{REFERENCES}

1. Sandu BK et al JPGN 2010:50:S1-S13.

2. Whiting $P$ et al. http://.biomedcentral.com/1471-2288/3/25 